\title{
Normalized differential method for improving the signal-to-noise ratio of distributed acoustic sensor
}

\author{
ISlam Ashry, ${ }^{1,3}$ YuAn MaO, ${ }^{1,3}$ Mohd Sharizal Alias, ${ }^{1}$ TIEn KHEE NG, ${ }^{1}$ \\ Frode Hveding, ${ }^{2, \#}$ Muhammad ArSalan, ${ }^{2}$ and Boon S. Ool ${ }^{1, *}$ \\ ${ }^{1}$ King Abdullah University of Science and Technology (KAUST), Computer, Electrical, and Mathematical Sciences \\ and Engineering, Thuwal 23955-6900, Saudi Arabia \\ ${ }^{2}$ EXPEC Advanced Research Center, Saudi Aramco, Dhahran, Saudi Arabia \\ ${ }^{3}$ These authors contributed equally to this work \\ \#rode.hveding@aramco.com,*boon.ooi@kaust.edu.sa
}

\begin{abstract}
We experimentally introduce a normalized differential method to enhance the time domain signal-to-noise ratio (SNR) of optical fiber distributed acoustic sensor (DAS). The reported method is calibrated against the typical differential method in noisy DAS systems, including those utilizing a relatively wide linewidth laser or few-mode fiber (FMF). In these two systems, the normalized differential method respectively identifies the position information of various vibration events with $1.7 \mathrm{~dB}$ and $0.53 \mathrm{~dB}$ SNR improvement. We further demonstrate the ability to locate positions along a fiber that are subjected to vibrations of frequencies higher than that the theoretical maximum, but without determining these frequencies.
\end{abstract}

(C) 2019 Optical Society of America under the terms of the OSA Open Access Publishing Agreement

\section{Introduction}

Among existing approaches for sensing, the fiber optic paradigm is particularly unique, given its numerous advantages including, immunity to electromagnetic interference, miniature sizes, harsh environment operation, and capability of large-scale multiplexing [1,2]. One potential example is the optical fiber distributed acoustic sensor (DAS) designed using the phasesensitive optical time domain reflectometry ( $\Phi$-OTDR) [3]. The $\Phi$-OTDR based DAS has been deployed in many applications such as real-time structural health monitoring [4], oil and gas industry [5], and aerospace transportation [6]. Its operation principle consists of launching a train of optical pulses, generated by a narrow linewidth laser, into a fiber under test (FUT). At the FUT input port, consecutive Rayleigh backscattered traces are recorded in the time domain. Each Rayleigh trace has a speckle-like profile because of coherent interference of the signals reflected by scattering centers within the injected pulse duration [3]. In the absence of intrusion along the FUT, i.e., no refractive index perturbation, the recorded Rayleigh traces are ideally identical. In case of an acoustic signal is applied at a position along the FUT, the effective refractive index changes at this position and consequently, the intrusion could be sensed by observing the intensity fluctuations of its corresponding speckle in the recorded traces.

In the typical direct and coherent detection schemes used for the $\Phi$-OTDR based DAS, there are several noise sources such as temporal pulse intensity fluctuation, laser phase noise and frequency drift, low extinction ratio of the launched pulses, and photodetector thermal and shot noise [2,3]. These noises degrade the stability of the recorded Rayleigh traces in the time-domain which consequently decreases the signal-to-noise ratio (SNR) of the system. Averaging traces is the common signal processing algorithm used to mitigate the intensity fluctuations of the Rayleigh traces. Unfortunately, this algorithm limits the maximum detectable vibrations frequency. In particular, assuming each averaged trace is calculated using $N$ consecutive traces, the effective sampling rate reduces to equal the repetition rate of 
injecting pulses divided by $N$. As a result, based on the sampling theory, the maximum detectable frequency (Nyquist frequency) is shrunk down by a factor of $N$, in comparison with the no averaging case. Instead, Yuelan Lu et al. introduce a moving averaging and moving differential algorithm to get around twice frequency response over that of the traditional averaging method [7], which is still significantly less than the Nyquist frequency. Other techniques such as wavelet denoising [8], bilateral filtering [9], and machine learning [10] are also used for SNR improvement; however, they are complicated to implement. Consequently, there is a strong motivation to develop a simple and effective signal processing method for enhancing the SNR of $\Phi$-OTDR based DAS.

We here introduce a straightforward signal processing technique to improve the time domain SNR of $\Phi$-OTDR based DAS system. In this method, we apply a normalized differential between consecutive Rayleigh traces without averaging. The capabilities of our method are experimentally investigated using the DAS direct detection scheme. The effectiveness of the normalized differential method is evaluated via comparing it with the standard differential method when using noisy optical components, such as comparatively wide linewidth laser or few-mode fiber (FMF). Using the reported signal processing technique, we can sense vibrations positions in these noisy DAS systems with enhanced SNR values. Additionally, we test the performance of our method when applying vibrations with frequencies beyond the Nyquist limit. This is to show the ability of our reported signal processing method to only identify the location information of these vibrations, but without calculating their frequencies which are higher than the theoretically maximum detectable limit (Nyquist frequency).

\section{Normalized differential method}

In this section, we define the normalized differential method and experimentally present its advantages over the typical differential one commonly used in the literature [7]. The used experimental setup of the $\Phi$-OTDR based DAS with direct detection is shown in Fig. 1. In this work, we use two different laser sources denoted as laser1 (JDSU, MTLG-A1) and laser2 (NKT Photonics, 15E) such that the linewidth of laser1 (laser2) equals $100 \mathrm{kHz}(100 \mathrm{~Hz})$. As the laser linewidth increases, its corresponding phase noise introduces more degradation to the SNR of DAS system [3]. In the experimental setup shown in Fig. 1, we use laser1 to generate continuous-wave (CW) light of $\sim 10 \mathrm{~mW}$ optical power at $1535 \mathrm{~nm}$ operation wavelength. The laser light is modulated by an electro-optic modulator (EOM1) (Covega, Mach-40085) driven by a pulse generator to produce optical pulses with $20 \mathrm{kHz}$ repetition rate and $100 \mathrm{~ns}$ width which offers $10 \mathrm{~m}$ sensing spatial resolution. Since the EOM1 is sensitive to the input light polarization state, we use a polarization controller (PC1) to maximize the peak output power of the generated pulses by EOM1. To enhance the extinction ratio (ER) of the optical pulses, another electro-optic modulator (EOM2) (Covega, Mach-10 063) is synchronized with the EOM1 through the pulse generator [11]. Again, a polarization controller (PC2) tunes the peak power of the pulses produced by EOM2 to its maximum value. Then the modulated light is amplified by an erbium-doped-fiber-amplifier (EDFA1) to

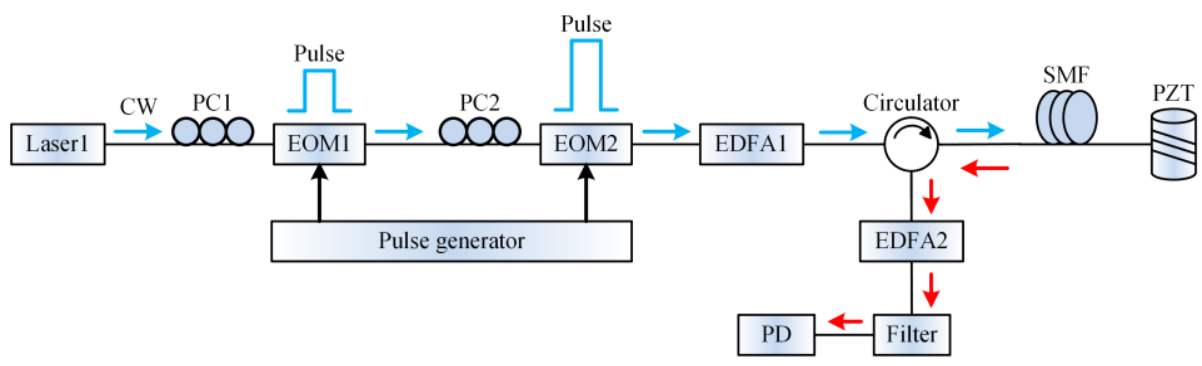

Fig. 1. Experimental setup of the $\Phi$-OTDR based DAS with direct detection. 
be launched through a circulator into a single-mode fiber (SMF) of $4 \mathrm{~km}$ length. A $10 \mathrm{~m}$ section of the SMF near its end is attached to a piezoelectric transducer (PZT) cylinder (APC International, 42-1091) such that its vibrations frequency is controllable via a function generator. The Rayleigh backscattered light from the SMF is amplified by another erbiumdoped-fiber-amplifier (EDFA2) and its amplified spontaneous emission (ASE) noise is filtered out by a fiber Bragg grating. Finally, the backscattered traces are recorded by a photodetector (PD) (Thorlabs, PDB 470C) and sampled at $125 \mathrm{MHz}$ rate using an oscilloscope (Tektronix, DPO70404C).

We first carry out the experiment when keeping the PZT static. Figure 2(a) shows a representative example of 100 consecutive recorded Rayleigh traces. For each trace, as shown in Fig. 2(a), the backscattered signals from the fiber have speckles of different intensities caused by the interference between the backscattered Rayleigh signals of different fiber's scattering centers within the injected pulse width [3]. We do not have control on the intensities of these speckles because their interference relies on the random orientations of the fiber's scattering centers, polarization and phase of the input optical pulses, etc. The highintensity signal at the beginning of the traces is caused by the fiber front facet reflection, not Rayleigh scattering. There are typically two targets for an $\Phi$-OTDR based DAS: discovering a location along the fiber subjected to an acoustic wave, and calculating the frequency components of these vibrations. In the literature, the first aim is normally attained via using the typical differential method, i.e., subtracting the consecutive Rayleigh traces [3,7]. A typical differential trace is defined as $\Delta R=\left(R_{i+1}-R_{i}\right), i \in[1, M-1]$, where $R_{i}$ denotes the $i$ th Rayleigh trace and $M$ is the total number of traces. In Fig. 2(b), we present the differential signal of the 100 consecutive traces. The results of Fig. 2(b) basically define the background noise of our experimental setup. Obviously, the noise values are not uniform along the fiber length; however, they depend on the original intensities within the Rayleigh traces. For more
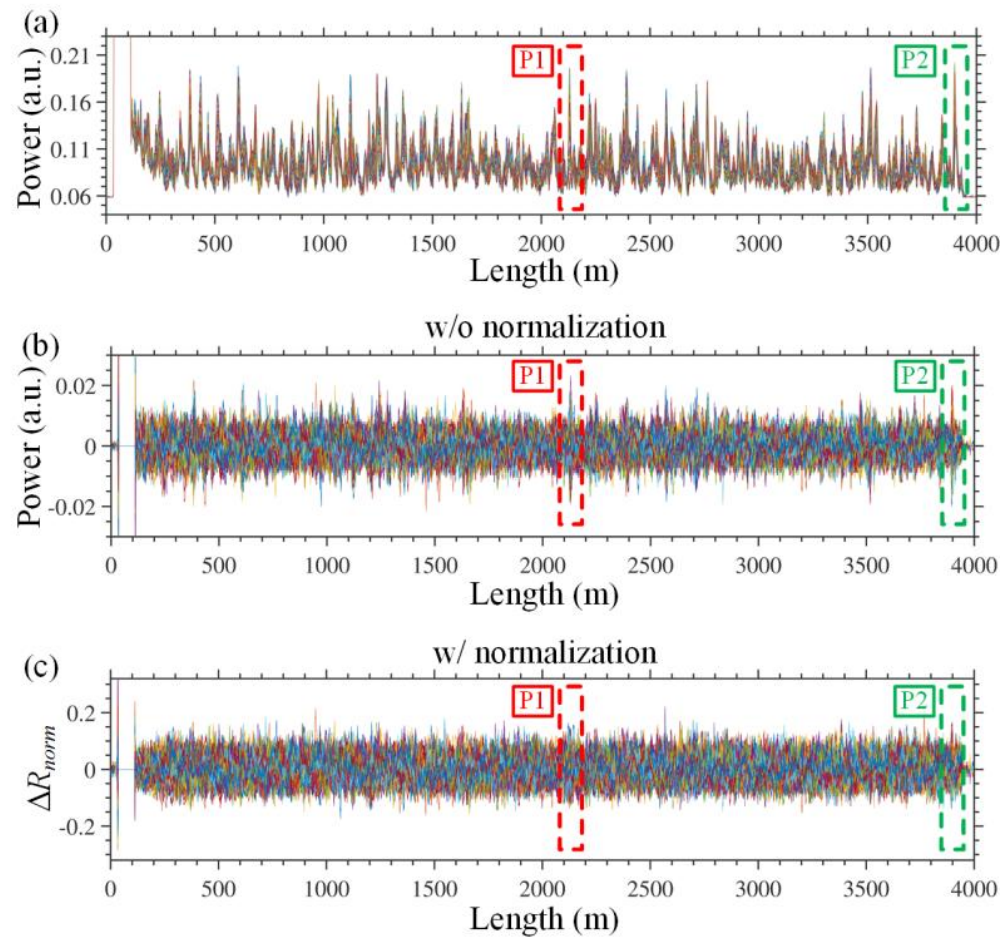

Fig. 2. (a) 100 consecutive Rayleigh traces recorded when the PZT is static. Difference between the 100 traces using the typical differential (b) and normalized differential method (c). 
clarification, we focus on the behavior of two arbitrary high-intensity speckles (marked as P1 and P2) in Fig. 2(a). By monitoring their corresponding noise values, as shown in Fig. 2(b), these two speckles produce higher noise values than those of lower Rayleigh intensities. This is to be expected since if assuming the percentage of trace fluctuations is on average constant along the whole fiber, the differences between high-intensity speckles should be larger than that of low-intensity ones. Considering the worst case scenario when a vibration event occurs at a position along the fiber which originally produces a low-intensity signal within the Rayleigh traces, the corresponding differential signal of this event could submerge into the noise background showing misleading to the vibration location. Instead, we here develop a normalized differential method that provides signals independent of the speckles intensities within the Rayleigh traces. We define the normalized differential trace as $\Delta R_{\text {norm }}=\left(R_{i+1}-R_{i}\right) / R_{i}$. Figure 2(c) shows $\Delta R_{\text {norm }}$ of the 100 Rayleigh traces presented in Fig. 2(a). In contrast to the results of Fig. 2(b), our method obviously improves the noise equalization along the fiber which consequently would enable figuring out vibrations locations even if their Rayleigh signal is originally weak.

\section{Detection of PZT vibrations}

To investigate the influence of the reported normalized differential method on the SNR of the DAS system, we compare it with the typical differential method when detecting vibrations produced by the PZT, shown in Fig. 1. Figure 3(a) shows 100 consecutive Rayleigh traces recorded when the PZT, located near the SMF end, vibrates sinusoidally with $2 \mathrm{kHz}$ frequency. The end facet reflection of the optical fiber is intentionally suppressed via using the fiber bending-loss at its end. It is worth mentioning that these traces are raw without any averaging. In Figs. 3(b) and 3(c), we present the typical and normalized differential signals, respectively. The SNR is defined as the ratio between the peak-to-peak power variation at the vibrations location and that of the background noise level [7]. Following this definition, the SNR values of the signals shown in Figs. 3(b) and 3(c) are respectively $0.61 \mathrm{~dB}$ and $2.31 \mathrm{~dB}$.
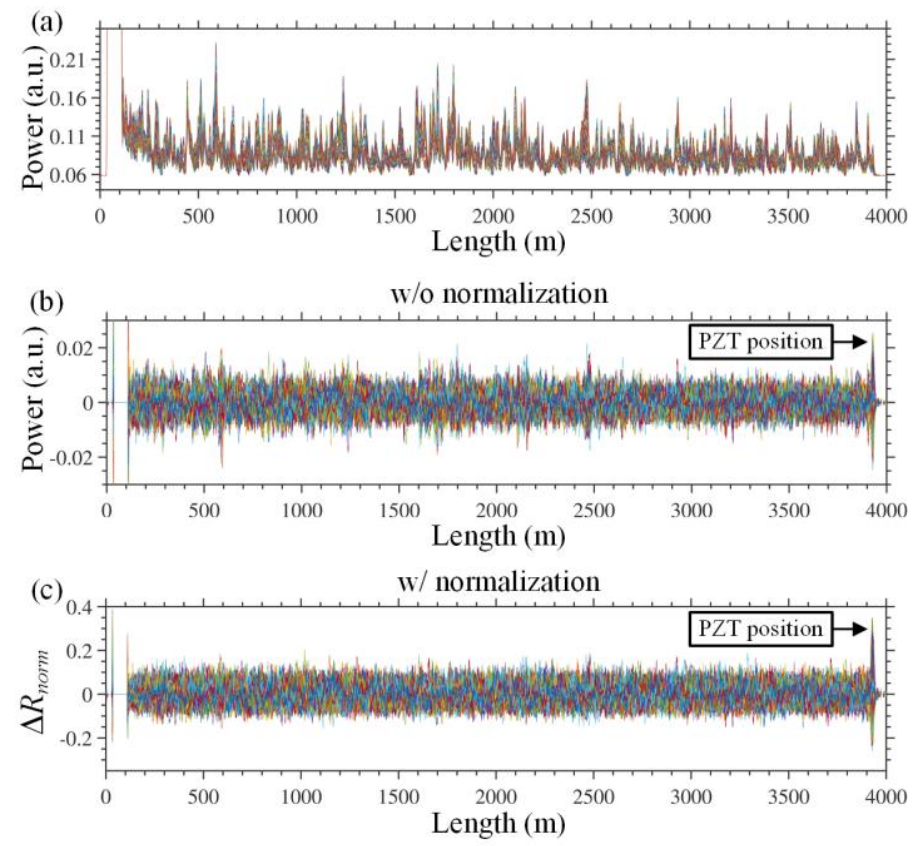

Fig. 3. (a) 100 consecutive Rayleigh traces recorded when the PZT is vibrating with $2 \mathrm{kHz}$ frequency. Difference between the 100 traces using the typical differential (b) and normalized differential method (c). 
Clearly, our signal processing method significantly improves the SNR of the $\Phi$-OTDR based DAS system. For more clarification, Figs. 4(a) and 4(b) show the temporal evolutions of the typical and normalized differential signal recorded within $5 \mathrm{~ms}$, respectively. The minimum acceptable SNR value of the $\Phi$-OTDR based DAS system is $2 \mathrm{~dB}$ [7]. Therefore, the vibrations signal is buried in noise in Fig. 4(a), while it is quite clear in Fig. 4(b).
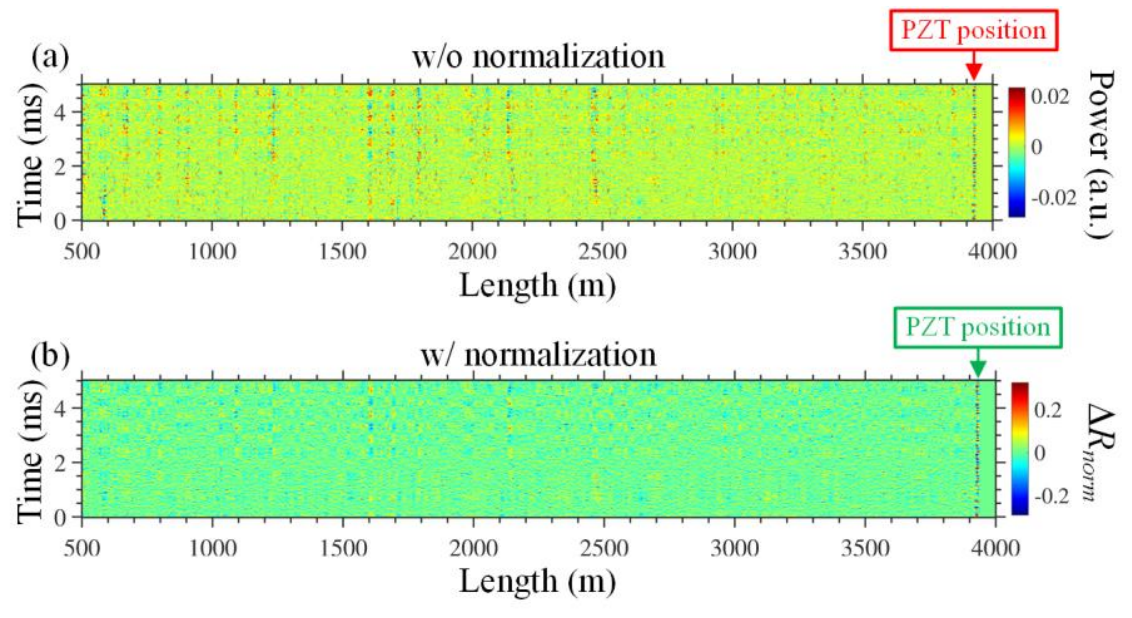

Fig. 4. Temporal evolution of the difference between the Rayleigh traces without (a) and with normalization (b).

\section{Detection of Rayleigh signals produced by a FMF}

Following our discussion in the introduction section regarding the operation principle of the $\Phi-O T D R$ based DAS, one can conclude that SMF is the optimum fiber choice for such sensing systems. This is because the speckles found in a Rayleigh trace, as those shown in Fig. 2(a), are produced by the light interference within the pump pulse width. In case if the used optical fiber supports propagating a significant number of modes, similar to the multimode fiber case, each spatial mode possesses its own independent interference which results in creating Rayleigh signals almost insensitive to vibrations. However, there is a motivation to use FMFs in DAS system because they support higher threshold power of nonlinearity, in comparison with the standard SMF, which would enable injecting pump pulses with higher peak-power [12]. This advantage can offer DAS with longer sensing range and higher spatial resolution. However, the inevitable intermodal coupling in such specialty fiber is considered a noise source [13].

In this section, we test the performance of our signal processing method when using the relatively noisy FMF-based DAS system. In the experimental setup shown in Fig. 1, we use laser2 instead of laser1 and we replace the SMF with a $\sim 1 \mathrm{~km} \mathrm{FMF} \mathrm{(YOFC} \mathrm{Co.,} \mathrm{Ltd.)} \mathrm{that}$ supports propagating the $\mathrm{LP}_{01}, \mathrm{LP}_{11 \mathrm{a}}$, and $\mathrm{LP}_{11 \mathrm{~b}}$ mode at the $1535 \mathrm{~nm}$ operation wavelength. We here use the FMF as a noise source instead of the relatively wide linewidth laser. This is mainly to assess the effectiveness of the normalized signal processing method with the potentially diverse noise sources in the DAS system. To make this experiment more challenging, we detect vibrations produced by a pencil break event [Fig. 5(a)] instead of using the PZT cylinder which is attached directly to the fiber. In particular, just before the FMF end, we stick a $10 \mathrm{~m}$ fiber loops to an aluminum plate of $1 \mathrm{~mm}$ thickness. As shown in Fig. 5(a), we break the pencil at a $\sim 3 \mathrm{~cm}$ distance from the FMF loops. Pencil break vibrations are the typical way to mimic the cracks sounds which might be produced in structural buildings [7]. The typical and normalized differential signal of the pencil break event are respectively shown in Figs. 5(b) and 5(c). In these two figures, without averaging, we use the Rayleigh traces separated by ten steps $\left(R_{i+10}, R_{i}\right)$ rather than the consecutive ones. This is 
because although the pencil break event generates vibrations of frequencies within the range [0,5 MHz], the vibrations frequencies of the used aluminum plate cannot exceed $\sim 1 \mathrm{kHz}$ [7]. Consequently, the differences between the consecutive Rayleigh traces are very small to be detected, at the position where such low frequencies vibrations occur. As demonstrated in Figs. 5(b) and 5(c), the vibrations can be well detected using the two different signal processing methods. This is mainly because laser 2 significantly mitigates the system noise since its linewidth is much narrower than that of laser1. The SNR values in Figs. 5(b) and $5(\mathrm{c})$ are respectively $4.97 \mathrm{~dB}$ and $5.50 \mathrm{~dB}$ which indicate our reported signal processing method still outweighs the conventional one even in DAS systems of low noises. It is worth mentioning that the two high-value signals at the beginning of the fiber length in Figs. 5(b) and 5(c) are the result of subtracting the two edges of the front-facet reflection pulse. In other words, these two high-value signals are not linked to the Rayleigh scattering.

For comparison purposes, the normalized differential method respectively provides $1.7 \mathrm{~dB}$ and $0.53 \mathrm{~dB}$ SNR improvement for the experimental setups described in Section 3 and 4. This difference is mainly because the noise produced by the FMF and laser2 is less than that produced by the SMF and laser1. The FMF is selected to have a step-index profile and we excite only the fundamental mode within the FMF such that the FMF operates in a quasisingle-mode (QSM) state. The QSM operation is realized by perfectly aligning the two axes of the circulator's SMF and the TMF, followed by joining them through fusion splicing [Fig. 5(d)]. The step-index profile of the FMF along with its operation in the QSM state mitigate intermodal coupling within the FMF [14]. Consequently, the noise produced by the narrower linewidth laser2 and the QSM-operated FMF is much less than the noise produced by the broader linewidth laser1 and the SMF. As a result, the reported signal processing method is more helpful for the more noisy system described in Section 3.

It is also worth mentioning that we here intentionally use the FMF instead of the SMF to increase the noise level within the DAS system. This is to prove the ability of our signal processing method to enhance the SNR of such noisy systems. However, the reported signal processing method is general and applicable for any type of optical fibers, such as SMF and FMF, and any vibration event (PZT, pencil break, loudspeaker, etc.).
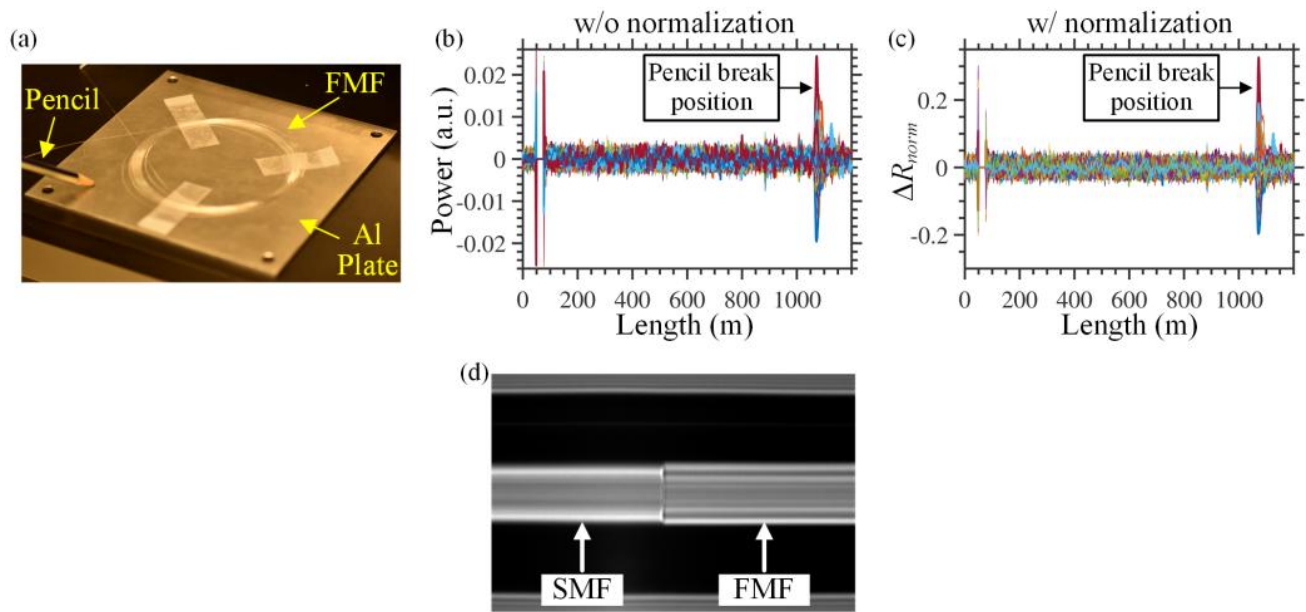

Fig. 5. (a) Experimental configuration of the pencil break sensing using a FMF. Difference between 100 traces, separated by a step equals 10 , using the typical differential (b) and normalized differential method (c). (d) Fusion splicing of the SMF and FMF, after aligning their axes.

\section{Discussion}

The normalized differential signal processing technique is mainly responsible for enhancing the time domain SNR of the DAS system. In other words, it improves identifying a position 
along the FUT subjected to vibrations. On the other hand, there is another major function of DAS system which is calculating the frequency components of the vibrations. This can be calculated by applying the fast Fourier transform to the Rayleigh raw traces at the vibrations position. It is worth mentioning that the latter DAS function cannot be enhanced by the normalized differential method. However, the reported method should always improve the time domain SNR in spite of the vibrations frequency. Since the repetition rate we use in our experiments is $20 \mathrm{kHz}$ and following the sampling theory, the maximum detectable vibrations frequency in our system is $10 \mathrm{kHz}$ (Nyquist frequency). We proceed to investigate the capability of the normalized differential method to sense locations along the FUT where vibrations occur with the Nyquist frequency and beyond. Since we can accurately control the PZT vibrations frequency, we use the experimental setup shown in Fig. 1 such that the PZT is driven by $10 \mathrm{kHz}$ and $15 \mathrm{kHz}$. Figures $6(\mathrm{a})$ and $6(\mathrm{~b})$ respectively show the position information and the power spectrum of the $10 \mathrm{kHz}$ vibrations event. Similarly, Fig. 6(c) presents the position information of the $15 \mathrm{kHz}$ event but its vibrations frequency cannot be detected since it is beyond the Nyquist frequency. Clearly, our method is still applicable for discovering vibrations position information even beyond the Nyquist frequency.
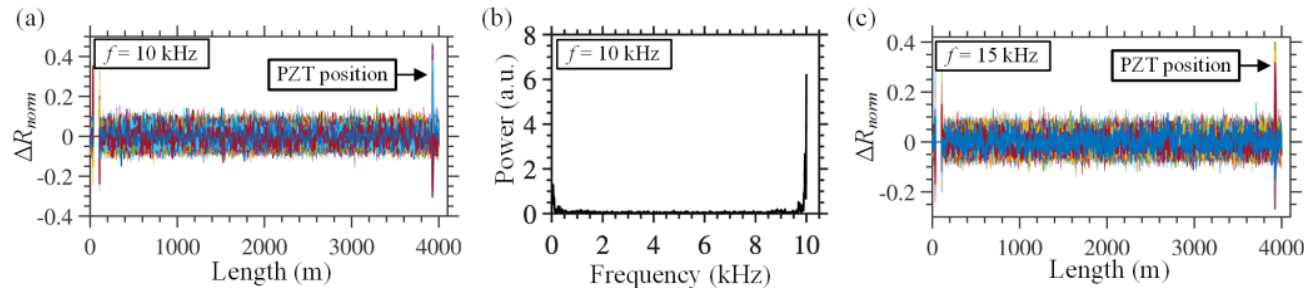

Fig. 6. (a) Normalized differential signals of $10 \mathrm{kHz}$ vibrations event, and (b) its corresponding power spectrum. (c) Normalized differential signals of $15 \mathrm{kHz}$ vibrations event.

Averaging Rayleigh traces can be combined with the reported normalized differential method for further improvement to the SNR of the DAS system. As a representative example, we average every two consecutive Rayleigh traces, and we then apply the normalized differential method, when the PZT cylinder is vibrating with a $2 \mathrm{kHz}$ frequency. Figures 7 (a) and 7(b) respectively show the typical and normalized differential signal of the $2 \mathrm{kHz}$ vibration event, after averaging. The SNR values in Figs. 7(a) and 7(b) are respectively 1.18 $\mathrm{dB}$ and $2.12 \mathrm{~dB}$ which indicate that our reported signal processing method provides $0.94 \mathrm{~dB}$ SNR improvement, when combined with the averaging method in this example.
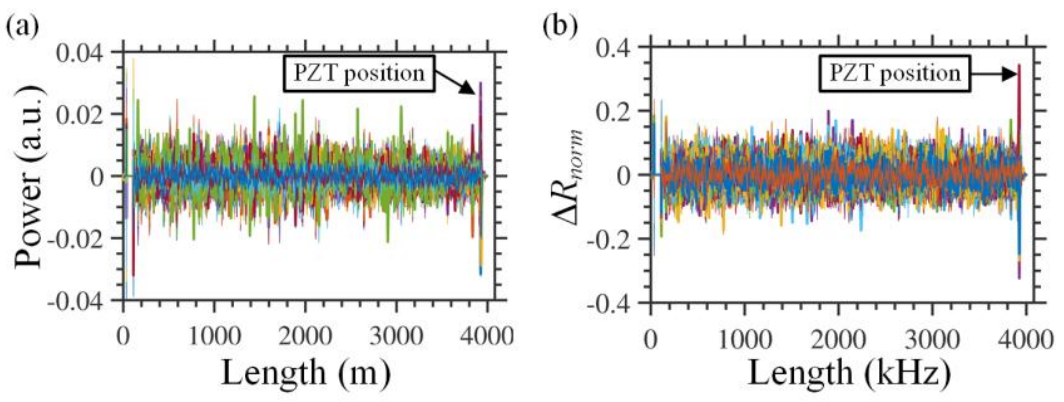

Fig. 7. Difference between 100 averaged traces using the typical differential (a) and normalized differential method (b).

\section{Conclusion}

In conclusion, we report the normalized differential method as a powerful tool for improving the performance of the $\Phi$-OTDR based DAS system. This technique equalizes the noise level along the entire FUT which results in enhanced time domain SNR of the DAS system. We 
compare the reported signal processing method with the typical differential one when analyzing the data produced by the relatively noisy DAS systems that include wide linewidth laser or FMF. In both systems, our method outperforms the conventional one in terms of discovering the vibrations position with high SNR. The introduced method is applicable when vibrations are with frequencies beyond the Nyquist limit.

Funding. Saudi Aramco (RGC/3/3138-01); King Abdullah University of Science and Technology (KAUST) baseline funding (BAS/1/1614-01-01); KAUST equipment funding (KCR/1/2081-01-01) and (GEN/1/6607-01-01).

\section{References}

1. B. Culshaw and A. Kersey, "Fiber-optic sensing: A historical perspective," J. Light. Technol. 26, 10641078 (2008).

2. X. Bao and L. Chen, "Recent Progress in Distributed Fiber Optic Sensors," Sensors (Switzerland) 12, 8601-8639 (2012).

3. X. Bao, D. P. Zhou, C. Baker, and L. Chen, "Recent Development in the Distributed Fiber Optic Acoustic and Ultrasonic Detection," J. Light. Technol. 35, 3256-3267 (2017).

4. J. C. Juarez and H. F. Taylor, "Field test of a distributed fiber-optic intrusion sensor system for long perimeters," Appl. Opt. 46, 1968 (2007).

5. T. Yamate, G. Fujisawa, and T. Ikegami, "Optical Sensors for the Exploration of Oil and Gas," J. Light. Technol. 35, 3538-3545 (2017).

6. S. Merlo, P. Malcovati, M. Norgia, A. Pesatori, C. Svelto, A. Pniov, A. Zhirnov, E. Nesterov, and V. Karassik, "Runways ground monitoring system by phase-sensitive optical-fiber OTDR," in 4th IEEE International Workshop on Metrology for AeroSpace, MetroAeroSpace 2017 - Proceedings (2017), pp. 523-529.

7. Y. Lu, T. Zhu, L. Chen, and X. Bao, "Distributed vibration sensor based on coherent detection of phaseOTDR," J. Light. Technol. 28, 3243-3249 (2010).

8. Z. Qin, L. Chen, and X. Bao, "Wavelet denoising method for improving detection performance of distributed vibration sensor," IEEE Photonics Technol. Lett. 24, 542-544 (2012).

9. H. He, L. Shao, H. Li, W. Pan, B. Luo, X. Zou, and L. Yan, "SNR enhancement in phase-sensitive OTDR with adaptive 2-D bilateral filtering algorithm," IEEE Photonics J. 9, 6802610 (2017).

10. J. Tejedor, J. Macias-Guarasa, H. Martins, J. Pastor-Graells, P. Corredera, and S. Martin-Lopez, "Machine Learning Methods for Pipeline Surveillance Systems Based on Distributed Acoustic Sensing: A Review," Appl. Sci. 7, 841 (2017).

11. M. Ren, D.-P. Zhou, L. Chen, and X. Bao, "Influence of finite extinction ratio on performance of phasesensitive optical time-domain reflectometry," Opt. Express 24, 13325 (2016).

12. M. Chen, A. Masoudi, F. Parmigiani, and G. Brambilla, "Distributed acoustic sensor based on a two-mode fiber," Opt. Express 26, 25399 (2018).

13. I. Ashry, A. Wang, and Y. Xu, "Mode-division-multiplexing of absorption-based fiber optical sensors," Opt. Express 24, 5186 (2016).

14. P. Sillard, M. Bigot-Astruc, and D. Molin, "Few-Mode Fibers for Mode-Division-Multiplexed Systems," J. Light. Technol. 32, 2824-2829 (2014). 